\title{
STATE-OF-THE-ART REVIEW ON APPLICATIONS OF HARMONY SEARCH META HEURISTIC ALGORITHM
}

\author{
Assif Assad \\ Department of Computer Science and Engineering, \\ Islamic University of Science and Technology, Awantipora, India
}

\begin{abstract}
Harmony Search (HS) a meta heuristic algorithm inspired by music improvisation process in which the musician searches for the best harmony and continues to polish the harmony in order to improve its aesthetics. The HS algorithm was introduced in the year 2001 and has found applications in diverse fields.

This manuscript reviews state-of-the-art applications of Harmony Search algorithm. As evidenced by a number of studies, this algorithm features several innovative aspects in its operational procedure that foster its utilization in diverse fields such as engineering, construction, telecommunications, robotics, health and energy
\end{abstract}

Keywords: Search, Evolutionary Algorithms, metaheuristic Optimization, Nature inspired Optimization.

Cite this Article: Assif Assad, State-of-the-Art Review on Applications of Harmony Search Meta Heuristic Algorithm, International Journal of Computer Engineering and Technology, 10(1), 2019, pp. 166-173.

http://iaeme.com/Home/issue/IJCET?Volume=10\&Issue $=1$

\section{INTRODUCTION}

Optimization is defined as the process of selecting the best option from a set of available alternatives. Every pro-cess has the potential to be optimized and many challenging problems in business, economics, science and engineering can be formulated as an optimization process. For example, the objective of formulated optimization problems can be the maximization of profit and/or quality, or minimization of time, cost and risk etc.

The application of optimization algorithms to real world problems has gained momentum in the last decade. Dating back to the early $1940 \mathrm{~s}$, diverse traditional mathematical methods such as linear programming (LP), nonlinear programming (NLP) or dynamic programming (DP) were first employed for solving complex optimization problems by resorting to di erent relaxation methods of the underlying formulation. These techniques are capable of cost-e ciently obtaining a global optimal solution in problem models subject to certain particularities 
(e.g. optimal sub structurability and subproblem overlap for dynamic programming), but unfortunately their application range does not cover the whole class of NP-complete problems, where an exact solution cannot be found in polynomial time. In fact, the solution space (and hence, the solving time) of the problem increases exponentially with the number of inputs, which makes them unfeasible for practical applications.

Many real life optimization problems are complex and thus difficult to solve in an exact manner within reasonable amount of time. The classical optimization methods have the limitation of being highly sensitive to the starting point and frequently converge to a local optimum. Classical optimization techniques can be broadly classified into two categories: direct search method and gradient based search method. In the direct search method, only the objective function and constraints are utilized for the search process, whereas in the gradient based search method, the first order and/or second order derivatives are utilized in the search process. Direct search methods have the limitation of slow convergence whereas gradient based search methods are faster however have the limitation of being not applicable to discontinuous and non di erentiable functions. Furthermore, both methods seek local optima, thus starting the search in the vicinity of a local optima causes them to miss the global optima.

Metaheuristic algorithms eradicate some of the afore mentioned defficulties and are quickly replacing the classical methods in solving complex nonlinear optimization problems. Metaheuristic algorithms typically intend to find a reasonably good solution close to optimization in reasonable amount of computational time. During the last few decades, several metaheuristic algorithms have been proposed including Genetic Algorithms, Genetic Programming, Evolutionary Programming, Evolutionary Strategies (ES), Differential Evolution, Ant Colony Optimization and Particle Swarm Optimization to name a few.

The Harmony Search (HS) algorithm is the musicians inspired metaheuristic algorithm developed by Geem et.al. in 2001 [1] and has found applications in diverse fields. Weyland [2] raised an issue regarding the novelty of Harmony Search algorithm by declaring it a special case of ES, however the pitch adjustment operator used in HS is entirely di erent than the mutation operator used in ES further HS utilizes the pitch adjustment operator (local search) probabilistically determined by PAR and thus the two cannot be considered same. Ample evidence has been provided by Saka et.al. in [3] to show HS is not a special case of ES even though superficially they seem to be identical.

\section{HARMONY SEARCH}

Harmony Search is a musicians behavior inspired evolutionary algorithm developed in 2001 by Geem et al.[1], though it is a relatively new meta heuristic algorithm, its effectiveness and advantages have been demonstrated in various applications like design of municipal water distribution networks [4], structural design [5], trafic routing [6], load dispatch problem in electrical engineering [7], multi objective optimization [8], rostering problems [9], clustering [10], classification and feature selection $[11,12]$ to name a few. A detailed survey on applications of HS can be found in $[13,15]$. In order to explain the Harmony Search in detail, let us first idealize the improvisation process by a skilled musician. When a musician is improvising there are three possible choices:

1. Play any piece of music exactly from his memory.

2. Play something similar to a known piece.

3. Compose new or random notes.

Geem et al. [1] formalized these three options into quantitative optimization process and the three corresponding components become usage of harmony memory (HM), pitch adjusting, and randomization. The usage of HM is similar to the choice of the best fit 
individuals in genetic algorithms. In order to use this memory effectively, it is typically assigned a parameter called harmony memory considering rate (HMCR $2[0,1])$. If this rate is low (near 0 ), only few best harmonies are utilized and thus convergence of algorithm is slow. If this rate is very high (near 1), it results in exploitation of the harmonies in the HM, thus the solution space is not explored properly leading to potentially inefficient solutions. Typically HMCR $2[.7, .95]$ is used. The second component is pitch adjustment determined by a pitch bandwidth (BW) (also referred as fret width [15] ) and a pitch adjusting rate (PAR), it corresponds to generating a slightly di erent solution in the HS algorithm. Pitch can be adjusted linearly or nonlinearly however most often linear adjustment is used. So we have

$$
\mathrm{H}_{\mathrm{i}}^{\text {new }}=\mathrm{H}_{\mathrm{i}}^{\text {old }}+\mathrm{BW} \times \mathrm{r}_{\mathrm{i}} \text { where } \mathrm{r}_{\mathrm{i}} \in[1,1] \text { and } 1 \leq \mathrm{i} \leq \mathrm{D}
$$

Where $\mathrm{H}_{\mathrm{i}}{ }^{\text {old }}$ is the $\mathrm{i}^{\text {th }}$ component of the existing harmony or solution and $\mathrm{H}_{\mathrm{i}}{ }^{\text {new }}$ is the $\mathrm{i}^{\text {th }}$ component of new harmony after the pitch adjusting action and BW is the bandwidth. The Equation (1) essentially produces a new solution around the existing solution by altering it slightly by a very small random amount. Here $r_{i}$ is a random number generated in the range of $[-1,1]$ and $\mathrm{D}$ is total number of components in the harmony. The pitch adjusting rate (PAR) controls the degree of adjustment. A low pitch adjusting rate with a narrow bandwidth can slow down the convergence of HS because of limitation in exploration of only a small subspace of the whole search space. On the other extreme a very high PAR with a wide bandwidth may cause the algorithm to swing around some optimal solution. Thus the recommended value of PAR is $[.1, .5]$. The third component of the HS is the randomization, which is used to increase the exploration of the search space. Although pitch adjustment plays a somewhat similar role, but it is confined to close neighborhood of harmony and thus corresponds to local search. The use of randomization pushes the algorithm further to explore diverse search areas to find the global optima. The pseudo code of harmony search is shown as Algorithm 1. In the pseudo code $\mathrm{H}$ represents a potential solution or harmony, rand $[0,1]$ is a uniformly distributed random number generator, rand_int(1,HMS) generates a uniformly distributed integer random number between 1 and HMS, size of harmony memory is represented as HMS and D is the dimension of problem.

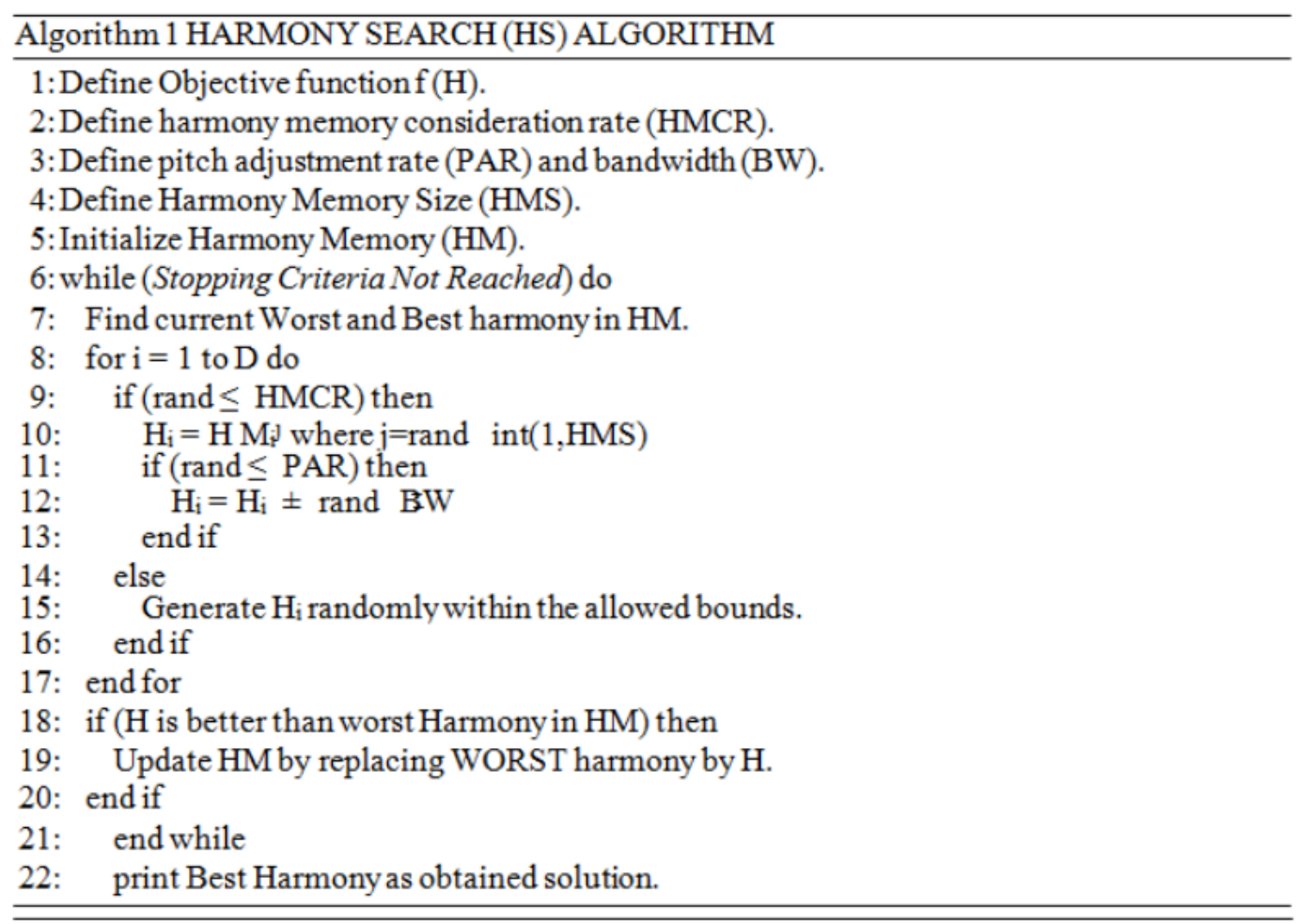




\subsection{Overview of Harmony Search Applications}

Sudoku is a Japanese puzzle which consists of an $\mathrm{N} \times \mathrm{N}$ square that is divided into $\sqrt{\mathrm{N}}$ subsquares, each of size $\sqrt{\mathrm{N}} \times \sqrt{\mathrm{N}}$. Here $\mathrm{N}$ is a perfect square and is known as the order of the Sudoku. In the beginning, there are some static numbers (called givens or fixed) in the puzzle. The game is to fill all non-givens such that each row, column and sub-square contains each integer from 1 to N exactly once. Sudoku is a well-known NP complete problem [16]. Figure 1 is an example of a Sudoku puzzle of order 9 and Figure 2 represents its solution. In the solution, each row, column and sub-square contains integers from 1 to 9 exactly once, further the givens remain intact.

Sudoku is linked to real world applications including conflict free wavelength routing in wide band optical networks, statistical design and error correcting codes, as well as timetabling and experimental design [17]. Another closely related problem to sudoku is generating threshold matrix for halftoning grayscale images [18]. In [19] Assad and Deep explored the hybridization between Harmony Search and Hill Climbing algorithm by utilizing the exploration power of the former and exploitation power of the latter in the context of solving Sudoku which is a well-known hard Combinatorial Optimization problem. The hybrid algorithm is called Harmony Search Hill Climber (HSHC). In order to extend the exploration capabilities of HSHC it is further modified to create three di erent algorithms namely Retrievable Harmony Search Hill Climber (RHSHC), Global Best Retrievable Harmony Search Hill Climber (GB-RHSHC) and Random Best Retrievable Harmony Search Hill Climber (RB-RHSHC). A set of 25 puzzles (5 each of type Beginner, Easy, Medium, Hard and Expert) has been taken to test the e ectiveness of the proposed algorithms. Comparing the four proposed algorithms RHSHC outperforms its three variations in terms of e ectiveness. compar-ing RHSHC with the genetic algorithm [20] it has been concluded that former outperforms latter both in terms of e ectiveness and e ciency particularly for Hard and Expert level puzzles. Comparing RHSHC and hybrid AC3-tabu search algorithm [21] it has been concluded that RHSHC is very competent to hybrid AC3-tabu search algorithm.

Given a simple undirected graph $\mathrm{G}=(\mathrm{V} ; \mathrm{E})$ where $\mathrm{V}=\{1,2 \ldots \mathrm{N}\}$ is the vertex set and $\mathrm{E}=$ $\mathrm{V} \times \mathrm{V}$, is the edge set. A clique $\mathrm{C}$ is the complete sub graph of $\mathrm{G}$ i.e. all the vertices of $\mathrm{C}$ are pair wise adjacent. The size of clique is the number of vertices in it and the maximum clique is the one having maximum cardinality. MCP is one of the most studied combinatorial optimization problems because it has wide range of practical applications in numerous fields like coding theory, bioinformatics and chemo informatics, examination planning, scheduling, financial networks,

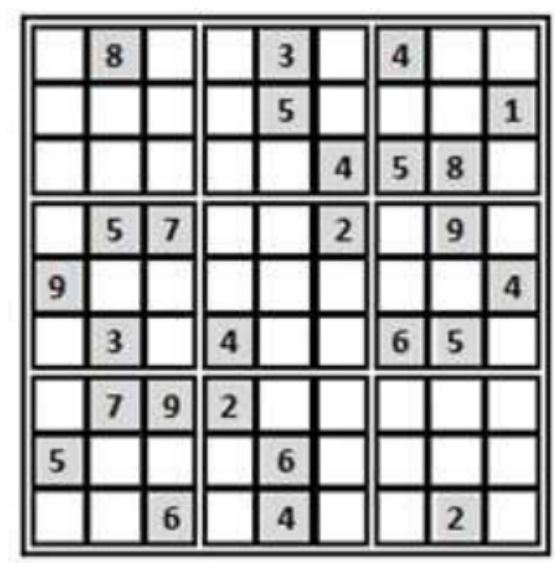

Figure 1 A Sudoku Puzzle with 26 givens. 


\begin{tabular}{||l|l|l||l|l|l|||l|l|l|}
\hline 7 & 8 & 5 & 9 & 3 & 1 & 4 & 6 & 2 \\
\hline 2 & 4 & 3 & 8 & 5 & 6 & 9 & 7 & 1 \\
\hline 6 & 9 & 1 & 7 & 2 & 4 & 5 & 8 & 3 \\
\hline \hline 4 & 5 & 7 & 6 & 1 & 2 & 3 & 9 & 8 \\
\hline 9 & 6 & 8 & 5 & 7 & 3 & 2 & 1 & 4 \\
\hline 1 & 3 & 2 & 4 & 9 & 8 & 6 & 5 & 7 \\
\hline \hline 3 & 7 & 9 & 2 & 8 & 5 & 1 & 4 & 6 \\
\hline 5 & 2 & 4 & 1 & 6 & 7 & 8 & 3 & 9 \\
\hline 8 & 1 & 6 & 3 & 4 & 9 & 7 & 2 & 5 \\
\hline
\end{tabular}

Figure 2 Solution of the Sudoku puzzle given in Figure 1.

social network analysis, signal transmission analysis, telecommunication and wireless networks, combinatorial auctions, visual feature matching, community detection, computer vision and information retrieval. In addition to these applications, the MCP is tightly related to some important combinatorial optimization problems such as clique partitioning, graph clustering, graph vertex coloring, max-min diversity, optimal winner determination, set packing and sum coloring [22]. These problems can either be directly formulated as a MCP or has a sub problem which requires to find a maximum clique. MCP is highly untractable and its decision version is among the first $21 \mathrm{NP}$-complete problems presented in Karp's seminal paper on computational complexity [23]. Even its approximation with in a constant factor are NP-Hard [24-28]. On the one hand, effective exact methods have been developed mainly based on the general branch and bound framework. These methods have the theoretical advantage of guaranteeing the optimality of the solution found. However, due to the inherent computational complexity of the MCP, exact methods can require a prohibitive computing time in general case and are often applicable to problems of small sizes. On the other hand, to handle problems whose optimal solutions cannot be reached within reasonable time using exact methods various heuristic and meta heuristic algorithms have been developed. Early attempts of using Genetic algorithms (GA) for MCP date back to 1990s. Other popular heuristics for the MCP include Simulated Annealing, Artificial Neural Networks, Ant Colony Optimization and Biomolecular Filtering.

Capabilities of Harmony Search (HS) algorithm for solving maximum clique problem has been investigated in

Two different instantiations of a generic HS algorithm namely (HS MCP) and (HSI MCP) for MCP has been proposed. HS MCP has better exploitation and inferior exploration capabilities than HSI MCP whereas HSI MCP has better exploration and inferior exploitation capabilities than HSI MCP, it has been concluded that former performs better than latter by testing them on all the instances of DIMACS benchmark graphs. HS MCP has been compared with a recently proposed Harmony search based algorithm for MCP called Binary Harmony search (BHS) and the simulation results show that HS MCP significantly outperforms BHS in terms of solution quality.

In [8] a new approach using Harmony Search (HS) algorithm is presented for placing Distributed Generators in radial distribution systems. The approach makes use of a multiple objective planning framework, named an Improved Multi-objective HS (IMOHS), to evaluate the impact of DG placement and sizing for an optimal development of the distribution system. The optimum sizes and locations of DG units are found by considering the power losses and voltage profile as objective functions. The feasibility of the proposed technique is 
demonstrated in two distribution networks, where the qualitative comparisons are made against a well-known technique, known as Non-dominated Sorting Genetic Algorithm II (NSGA-II).

In [28] a Harmony Search based algorithm called discrete harmony search (DHS) algorithm is used to solve the Flex-ible job-shop scheduling problem (FJSP). FJSP is a practically useful extension of the classical job shop scheduling problem. The objectives are the weighted combination of two minimization criteria namely, the maximum of the completion time and the mean of earliness and tardiness. Initially a new method is developed for the initial machine assignment task. Existing heuristics are employed for initializing the harmony memory with discrete machine permutation for machine assignment and job permutation for operation sequencing. Secondly a new rule is developed for the improvisation to produce a new harmony for FJSP incorporating machine assignment and operation sequencing. Finally several local search methods are embedded to enhance the local exploitation ability of the algorithm.

\section{CONCLUSION}

This article introduced the recent significant developments in the structure of Harmony Search algorithm and also describes its recent state-of-the-art applications. As evidenced by a number of studies, this algorithm features several innovative aspects in its operational procedure that foster its utilization in diverse fields such as engineering, construction, telecommunications, robotics, health and energy.

\section{REFERENCES}

[1] Z. W. Geem, J. H. Kim, and G. Loganathan, "A new heuristic optimization algorithm: harmony search," Simulation, vol. 76, no. 2, pp. 60-68, 2001.

[2] D. Weyland, "A rigorous analysis of the harmony search algorithm: how the research community can be," Modeling, Analysis, and Applications in Metaheuristic Computing: Advancements and Trends: Advancements and Trends, p. 72, 2012.

[3] M. Saka, O. Hasanc, ebi, and Z. Geem, "Metaheuristics in structural optimization and discussions on harmony search algorithm," Swarm and Evolutionary Computation, vol. 28, pp. 88-97, 2016.

[4] Z. W. Geem, "Optimal cost design of water distribution networks using harmony search," Engineering Optimization, vol. 38, no. 03, pp. 259-277, 2006.

[5] S. Gholizadeh and A. Barzegar, "Shape optimization of structures for frequency constraints by sequential harmony search algorithm," Engi-neering Optimization, vol. 45, no. 6, pp. 627-646, 2013.

[6] Z. W. Geem, K. S. Lee, and Y. Park, "Application of harmony search to vehicle routing," American Journal of Applied Sciences, vol. 2, no. 12,1552-1557, 2005.

[7] L. Wang and L.-p. Li, "An e ective di erential harmony search algorithm for the solving non-convex economic load dispatch problems," International Journal of Electrical Power \& Energy Systems, vol. 44, no. 1, pp. 832-843, 2013.

[8] K. Nekooei, M. M. Farsangi, H. Nezamabadi-Pour, and K. Y. Lee, “An improved multi-objective harmony search for optimal placement of dgs in distribution systems," Smart Grid, IEEE Transactions on, vol. 4, no. 1, pp. 557-567, 2013.

[9] M. Hadwan, M. Ayob, N. R. Sabar, and R. Qu, "A harmony search algorithm for nurse rostering problems," Information Sciences, vol. 233,126-140, 2013. 
[10] D. C. Hoang, P. Yadav, R. Kumar, and S. K. Panda, "Real-time implementation of a harmony search algorithm-based clustering protocol for energy-e cient wireless sensor networks," Industrial Informatics, IEEE Transactions on, vol. 10, no. 1, pp. 774-783, 2014.

[11] R. Diao and Q. Shen, "Feature selection with harmony search," Systems, Man, and Cybernetics, Part B: Cybernetics, IEEE Transactions on, vol. 42, no. 6, pp. 15091523, 2012.

[12] H. Fattahi, A. Gholami, M. S. Amiribakhtiar, and S. Moradi, "Estimation of asphaltene precipitation from titration data: a hybrid support vector regression with harmony search," Neural Computing and Applications, vol. 26, no. 4, pp. 789798, 2015.

[13] D. Manjarres, I. Landa-Torres, S. Gil-Lopez, J. Del Ser, M. N. Bilbao, S. SalcedoSanz, and Z. W. Geem, "A survey on applications of the harmony search algorithm," Engineering Applications of Artificial Intelligence, vol. 26, no. 8, pp. 1818-1831, 2013.

[14] A. Assad and K. Deep, "Applications of harmony search algorithm in data mining: A survey," in Proceedings of Fifth International Conference on Soft Computing for Problem Solving, pp. 863-874, Springer, 2016.

[15] Z. W. Geem and Y. Yoon, "Harmony search optimization of renewable energy charging with energy storage system," International Journal of Electrical Power \& Energy Systems, vol. 86, pp. 120-126, 2017.

[16] Y. Takayuki and S. Takahiro, "Complexity and completeness of finding another solution and its application to puzzles," IEICE transactions on fundamentals of electronics, communications and computer sciences, vol. 86, no. 5, pp. 1052-1060, 2003.

[17] S. K. Jones, P. A. Roach, and S. Perkins, "Construction of heuristics for a searchbased approach to solving sudoku," in Research and Development in Intelligent Systems XXIV, pp. 37-49, Springer, 2008.

[18] T. Mantere and J. Koljonen, "Solving and rating sudoku puzzles with genetic algorithms," in New Developments in Artificial Intelligence and the Semantic Web, Proceedings of the 12th Finnish Artificial Intelligence Conference STeP, pp. 86-92, Citeseer, 2006.

[19] A. Assad and K. Deep, "Harmony search based memetic algorithms for solving sudoku," International Journal of System Assurance Engi-neering and Management, pp. 1-14.

[20] K. N. Das, S. Bhatia, S. Puri, and K. Deep, "A retrievable ga for solving sudoku puzzles," tech. rep., Citeseer, 2012.

[21] R. Soto, B. Crawford, C. Galleguillos, E. Monfroy, and F. Paredes, "A hybrid ac3tabu search algorithm for solving sudoku puzzles," Expert Systems with Applications, vol. 40, no. 15, pp. 5817-5821, 2013.

[22] Q. Wu and J.-K. Hao, "A review on algorithms for maximum clique problems," European Journal of Operational Research, vol. 242, no. 3, pp. 693-709, 2015.

[23] R. M. Karp, Reducibility among combinatorial problems. Springer, 1972.

[24] U. Feige, S. Goldwasser, L. Lovasz,' S. Safra, and M. Szegedy, "Approximating clique is almost np-complete," 1991. 
[25] J. Håstad, "Clique is hard to approximate within $\mathrm{n}^{1}$," in Foundations of Computer Science, 1996. Proceedings., 37th Annual Symposium on, pp. 627-636, IEEE, 1996.

[26] N. E. Ordo'nez -Guillen' and I. M. Mart'inez-Perez,' "Heuristic search space generation for maximum clique problem inspired in biomolecular filtering," Journal of Signal Processing Systems, pp. 1-12, 2015.

[27] A. Assad and K. Deep, "A heuristic based harmony search algorithm for maximum clique problem," OPSEARCH, pp. 1-23, 2017.

[28] K.-Z. Gao, P. N. Suganthan, Q.-K. Pan, T. J. Chua, T. X. Cai, and C.-S. Chong, "Discrete harmony search algorithm for flexible job shop scheduling problem with multiple objectives," Journal of Intelligent Manufacturing, vol. 27, no. 2, pp. 363374, 2016. 